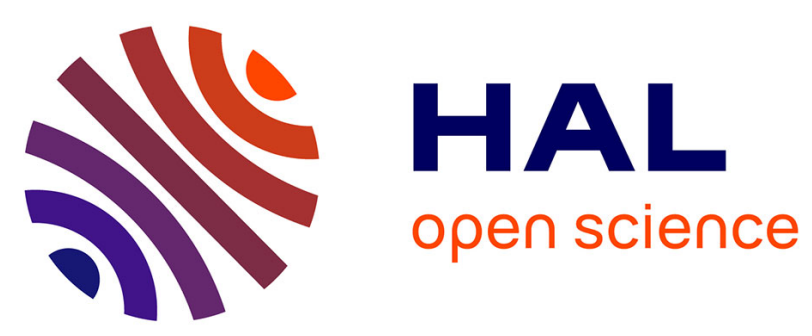

\title{
Incommensurate grain boundary in silicon and the silver-ratio sequence
}

Frédéric Lançon, Nina Gunkelmann, Damien Caliste, Jean-Luc Rouvière

\section{To cite this version:}

Frédéric Lançon, Nina Gunkelmann, Damien Caliste, Jean-Luc Rouvière. Incommensurate grain boundary in silicon and the silver-ratio sequence. Physical Review B: Condensed Matter and Materials Physics (1998-2015), 2019, 100 (11), 10.1103/PhysRevB.100.115307 . hal-02284426

\section{HAL Id: hal-02284426 https://hal.science/hal-02284426}

Submitted on 11 Sep 2019

HAL is a multi-disciplinary open access archive for the deposit and dissemination of scientific research documents, whether they are published or not. The documents may come from teaching and research institutions in France or abroad, or from public or private research centers.
L'archive ouverte pluridisciplinaire HAL, est destinée au dépôt et à la diffusion de documents scientifiques de niveau recherche, publiés ou non, émanant des établissements d'enseignement et de recherche français ou étrangers, des laboratoires publics ou privés. 


\title{
Incommensurate grain boundary in silicon and the silver-ratio sequence
}

\author{
Frédéric Lançon, ${ }^{1, *}$ Nina Gunkelmann, ${ }^{1,2}$ Damien Caliste,${ }^{1}$ and Jean-Luc Rouvière ${ }^{3, \dagger}$ \\ ${ }^{1}$ Univ. Grenoble Alpes, CEA, MEM, L_Sim, F-38000 Grenoble, France \\ ${ }^{2}$ Present address: Institute of Applied Mechanics, Technische Universität Clausthal, \\ Arnold-Sommerfeld-Straße 6, D-38678 Clausthal-Zellerfeld, Germany \\ ${ }^{3}$ Univ. Grenoble Alpes, CEA, MEM, Lemma, F-38000 Grenoble, France
}

(Dated: September 11, 2019)

\begin{abstract}
A scheme is proposed to solve the structure of incommensurate interfaces, starting from highresolution images of electron microscopy, supplemented by adapted simulation techniques and complemented by theoretical tools. Direct silicon bonding is a way to produce artificial interfaces, in particular incommensurate ones. We focus on a technology-driven tilt grain boundary in silicon. While the Fibonacci sequence, linked to the golden ratio, is a prototype of the quasicrystalline structures, a silver-ratio sequence allows us to analyze this incommensurate interface. The four-fold coordination of the $\mathrm{Si}$ atoms is kept at the interface.
\end{abstract}

\section{INTRODUCTION}

Direct wafer bonding ${ }^{1,2}$ is a pathway to produce grain boundaries that may not occur naturally. In particular, attractive structures for semiconductor technology can be produced by bonding together a $\operatorname{Si}\{011\}$ wafer and a $\mathrm{Si}\{100\}$ one, with the [001] direction of the first wafer aligned with the [110] direction of the second one ${ }^{3-7}$. Indeed, in this system with mixed orientations, the hole mobility and the electron mobility have opposite enhancement in the wafers, leading to important technological advancements ${ }^{8}$.

However, the resulting $90^{\circ}\langle 110\rangle$ tilt boundaries are incommensurate, since the two aligned directions [001] and [110] have periodicities with a ratio $\sqrt{2}$ between them. Because of this lack of periodicity of the grain boundaries, solving their atomic structures is not simple, though necessary to fully understand their properties. Several attempts have already been conducted ${ }^{5,9}$, but without considering their incommensurate nature.

Although more complex than the periodic cases, the theoretical background to describe incommensurate grain boundaries was quickly provided as an extension of the quasicrystal understanding ${ }^{10-15}$. However, nowadays, high-resolution electron microscopes, as well as mature and precise simulation techniques ${ }^{16-18}$, can be pursued to give accurate characterizations and quantitative analyses of the incommensurate grain boundaries.

In this work, we focus on the $90^{\circ}\langle 110\rangle$ tilt boundary, but the approach is general to incommensurate interfaces in covalent materials. Our purpose is not to completely explain the atomic structure of the $90^{\circ}\langle 110\rangle$ tilt grain boundary by energy considerations ${ }^{19}$, but rather to extract the local atomic structures from high-resolution experiments. Indeed, through pattern matching, the characteristics of the interface are highlighted and twodimensional coordinates are extracted. In principle, for a non periodic interface, a large area of the material should be analyzed to determine the subtle crystallinesite distortions at the interface. In our case however, two elemental units can be selected and arranged to produce infinite sequences relative to the silver ratio $1+\sqrt{2}$, thanks to the quasicrystalline framework (see section VI). From the 2D coordinates extracted from the experimental images of these units, we introduce a two-step energy minimization to retrieve the tridimensional atomic coordinates of the interface elements (see section IV). The atomic structures are then refined by nowadays precise DFT calculations (density functional theory).

Finally a complete atomic description of this incommensurate grain boundary in silicon is given. Note that a similar but metallic incommensurate boundary has been described with the same level of details ${ }^{20}$. However, we address here a different class of incommensurate grain boundaries where the structural unit model ${ }^{14,15}$ is well adapted to describe them.

\section{GRAIN-BOUNDARY DESCRIPTION}

A $90^{\circ}\langle 110\rangle$ tilt grain boundary in a cubic material is an interface between two crystalline grains having a common crystallographic direction $\langle 110\rangle$ but where one grain is rotated by $90^{\circ}$ with respect to the other one, around this common axis. Because the cubic cell of side $a$ is projected into a rectangle of sides $a$ and $\sqrt{2} a$ onto a $\{110\}$ plane, the two grains have no coincidence site lattice in this plane. If there was one, the site coordinates of this lattice expressed respectively in both $2 \mathrm{D}$ rectangular cells would imply relations $n a=m \sqrt{2} a$ with $n$ and $m$ being integers. The grain boundary is thus called incommensurate.

The orientation of the boundary itself is fixed by the grain bonding. In this study, the interface is parallel to the common axis and to one of the sides of the $2 \mathrm{D}$ rectangular cells (see figure 1). More precisely, $[1 \overline{1} 0]$ is the common axis of the two grains and taken as our $z$ axis. 


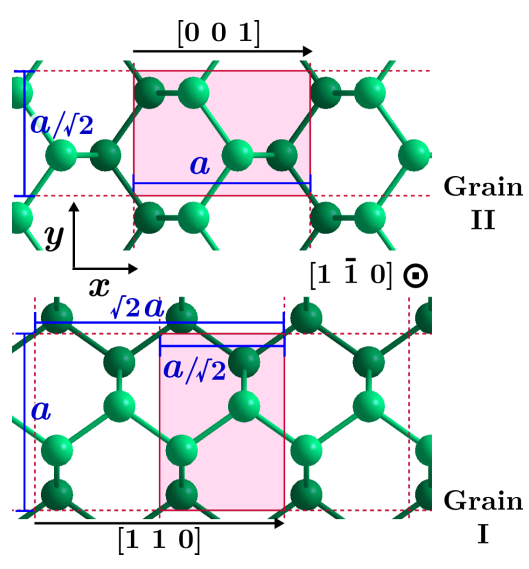

FIG. 1. Crystalline grain orientations on both side of the grain boundary. The Miller indices are relative to the cubic cell. The plan view is perpendicular to [1 10$]$, which is the common direction of both grains ( $z$-axes here). The shades of the spheres indicate their altitude $z=0$ or $z=a /(2 \sqrt{2})$. The light-shade rectangles of sides $a$ and $a / \sqrt{2}$ represent the silicon orthorhombic cells with the lattice vectors corresponding to [001], [110], and [1 $\overline{1} 0]$. The cubic cell is projected onto (1 $\overline{1} 0)$ as a rectangle of sides $a$ (side of the cube) and $\sqrt{2} a$ (diagonal of the face). The vector [001] of one of the crystal is parallel to the vector [110] of the other one, and thus the grain boundary is incommensurate. In this study, the interface is parallel to the $x z$-plane.

This axis is therefore a commensurate direction of the interface. The interface contains the [110] direction of one grain, let's say grain I, and thus contains the [001] direction of grain II. This direction is the incommensurate direction of the interface and is chosen to be our $x$ axis. The $y$ axes is perpendicular to the interface.

\section{APPROXIMANTS}

The periods of grains I and II along the incommensurate direction $(x)$ are denoted $s$ and $\ell$, respectively ( $s$ for short and $\ell$ for long). Grains I and II are, respectively, the lower and upper grains in figure 1 . In the ideal incommensurate grain boundary, $s=a / \sqrt{2}$ and $\ell=a$, where $a$ is the cubic lattice parameter of silicon. However, the atomic configurations considered for computation usually correspond to periodic approximants of the incommensurate grain boundary. In the same way that a sequence of rational numbers $n_{s} / n_{\ell}$ can tend to an irrational number, a sequence of periodic approximants can approach better and better an incommensurate structure. In the context of this article, a " $n_{s}: n_{\ell}$ approximant" is a periodic boundary where $n_{s}$ periodic cells of grain I are facing $n_{\ell}$ cells of grain II. The best matches between the two grains are obtained when the ideal lengths $n_{\ell} \ell$ and $n_{s} s$ have close values. This corresponds precisely to the strong condition to be verified by $n_{s}$ and $n_{\ell}$ for $n_{s} / n_{\ell}$ to be among the best rational approximations of the ideal

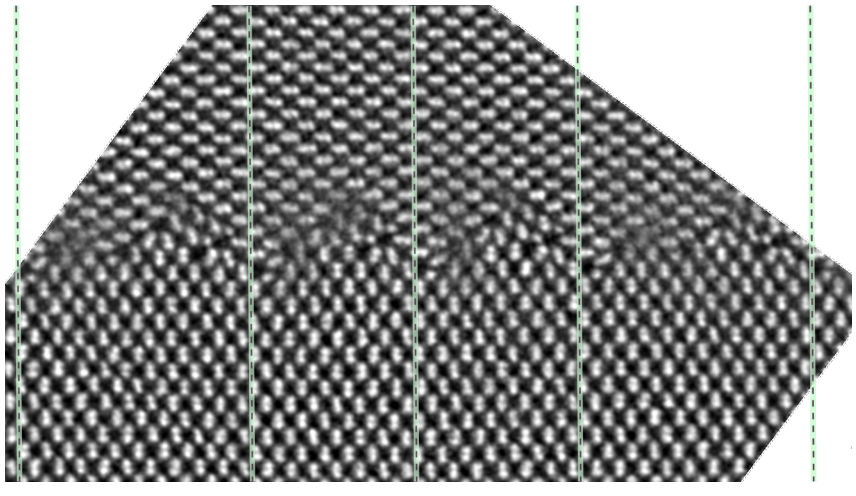

FIG. 2. Scanning transmission electron microscopy (STEM) image of the incommensurate grain boundary. Si atoms, individual or in adjacent pairs (dumbbells), are all resolved in this image. More precisely, each spot corresponds to an atomic column perpendicular to the plane view. Vertical lines indicate a possible decomposition into 7:5 and 10:7 approximants of the grain boundary (thinner and thicker strips, respectively). Note the good atomic match between atomic patterns at the different line locations making possible periodic boundary conditions for each approximant, or combinations of several 7:5 and 10:7 approximants.

value of $\ell / s$, i.e. $\sqrt{2}$ here:

$$
\Delta\left[n_{s}, n_{\ell}\right] \equiv\left|n_{\ell} \sqrt{2}-n_{s}\right|<|q \sqrt{2}-p|
$$

for any rational number $p / q$ so long as $q<n_{\ell}$. With this condition, the best approximations are the continuedfraction convergents of $\sqrt{2}$. These convergents are $1 / 1$, $3 / 2,7 / 5,17 / 12$, and more generally $p_{k} / q_{k}=\left(2 p_{k-1}+\right.$ $\left.p_{k-2}\right) /\left(2 q_{k-1}+q_{k-2}\right)$ at step $k>2$. In our calculations, the period $P$ of the $n_{s}: n_{\ell}$ approximant is the arithmetic mean of the corresponding ideal grain periods:

$$
P=a\left(n_{\ell}+n_{s} / \sqrt{2}\right) / 2
$$

The residual strains in grains I and II in the approximant are then respectively

$$
\begin{aligned}
\varepsilon_{x, I}=\left(P-n_{s} s\right) /\left(n_{s} s\right) & =\left(\frac{n_{\ell}}{n_{s}}-\frac{1}{\sqrt{2}}\right) / \sqrt{2} \\
\varepsilon_{x, I I}=\left(P-n_{\ell} \ell\right) /\left(n_{\ell} \ell\right) & =\left(\frac{n_{s}}{n_{\ell}}-\sqrt{2}\right) /(2 \sqrt{2}) \\
& =-\varepsilon_{x, I}+\mathcal{O}\left(\left(\frac{n_{s}}{n_{\ell}}-\sqrt{2}\right)^{2}\right)
\end{aligned}
$$

and thus the minimal strains are also obtained by the best rational approximations $n_{s} / n_{\ell}$ of $\sqrt{2}$. Note that a convergent $n_{s} / n_{\ell}$ of $\sqrt{2}$ corresponds to the convergent $n_{\ell} / n_{s}$ of $1 / \sqrt{2}$.

\section{ELECTRON MICROSCOPY RESULTS}

Figure 2 is an electron-microscopy (STEM) image of the grain boundary observed along the common [110] direction of the two grains. The experimental details are 
given in reference [5]. An atomistic model of the periodic 7:5 approximant of this grain boundary had already been proposed $^{5}$. Indeed, a repeating pattern can be recognized with a pseudo periodicity corresponding to $n_{\ell}=5$ periods of grain II and $n_{s}=7$ periods of grain I. However, even if $7 / 5$ is only $1 \%$ smaller than $\sqrt{2}$, this would correspond to a tensile strain in grain $\mathrm{I}$ and a compressive strain in grain II of $\pm 5 \times 10^{-3}$ (equation 3), impossible for the material to sustain macroscopically. In fact, a similar but longer pattern can also be identified in the microscopy images. It corresponds to a 10:7 approximant, i.e., $n_{s}=10$ and $n_{\ell}=7$. While $10 / 7$ does not belong to the best approximations of $\sqrt{2}$, this approximant has about the same strain amount than the 7:5 one, but with an opposite sign for each grain. Thus, combining them together, as in figure 2 , can reduce the strains to $\pm 9 \times 10^{-4}$. Indeed, $17 / 12=(7+10) /(5+7)$ is the next best rational approximation after $7 / 5$. In the following, we will consider the 7:5 and the 10:7 approximants as the structural units of the grain boundary. Their 3D structures will be solved by combining image analyses and numerical energy minimizations. The general way to combine them in a sequence of best approximants and thus to describe the infinite grain boundary will be discussed in section VI.

\section{FROM 2D TO 3D}

\section{Pattern recognition}

The resolution of the experimental images is high enough to deduce with a good precision the $x$ and $y$ atomic coordinates of the $\mathrm{Si}$ atomic columns perpendicular to the image plane. To automatically extract a large number of coordinates, a pattern recognition technique ${ }^{21}$ based on cross-correlation functions has been used. From the experimental image, three small portions representative of three material characteristics are selected: the individual atomic column and the two possible orientations of Si dumbbells composed of two columns. Three crosscorrelation functions are first calculated between the image and each of the three templates. As described in [21], three smoother patterns are obtained by averaging image sections located at high correlation maximums (see figure 3). A second set of correlation functions is then calculated. Taking advantage of the three-dimensional nature of the color image coding, we introduce here a way to gather these three functions and get a direct visualization of the information. For all pixels, each color component red, green, or blue, is associated with the value of one of the cross-correlation functions. Figure 3 shows that the different constituents of the grain boundary are now clearly identified. Then, isolated columns, or pairs of them, are associated with Gaussian functions that are fitted on the initial image using the locations of the correlation maxima as initial guesses. To improve the recognition of the atom positions, the low intensity

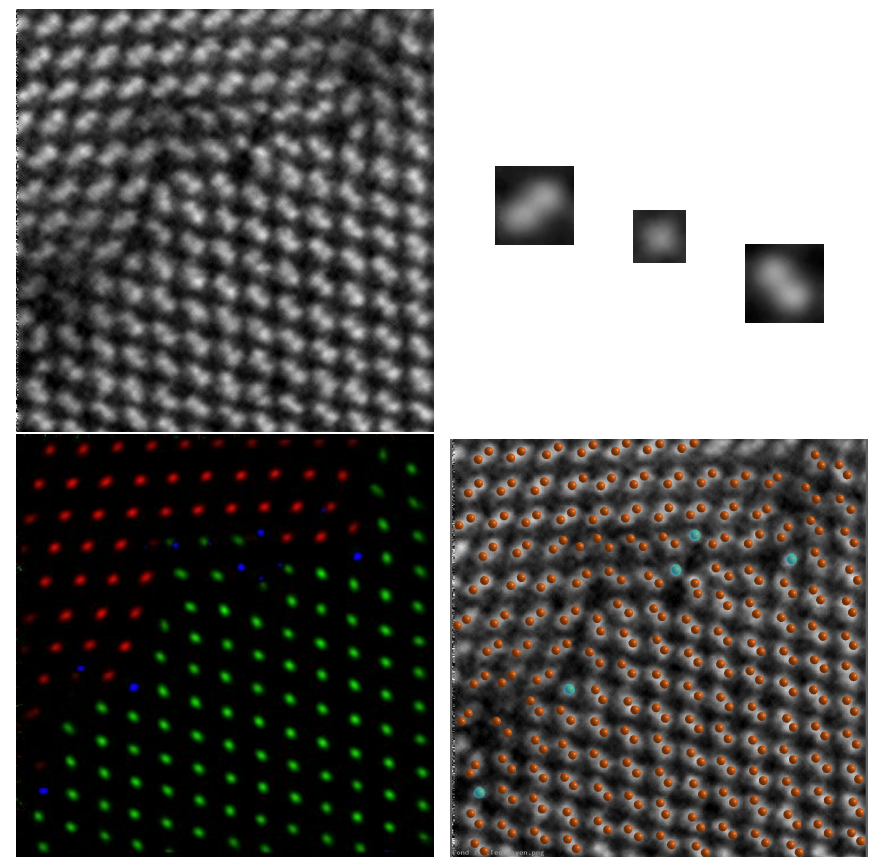

FIG. 3. (Color online) Illustration of the pattern recognition technique. (Top-left) Small part of an image, with grain II on top of grain I. (Top-right) Three patterns corresponding to grains I, II, and to isolated atomic columns. (Bottomleft) Color image representing the three correlation functions corresponding to the three patterns. Each correlation function represents one component of the RGB image coding (redgreen-blue). A gamma correction of 4 has been applied to enhance the highest values of the correlations, i.e., a power-law function with exponent 4 is applied to the components (normalized between 0 and 1). (Bottom-right) $x$ and $y$ atomic positions obtained by fitting, in the first image, individual or paired Gaussian functions, initially located at the maximum of the correlation functions. The individual atomic columns are distinguished by the color cyan.

around each image spot was assigned to zero thanks to an apodization function. The 2D Si positions finally extracted from the experimental image are also shown in Figure 3.

\section{Stillinger-Weber atomic interaction}

The 2D atomic coordinates, say $x$ and $y$, extracted from experimental images need to be completed by the $z$ coordinates, corresponding to the hidden component perpendicular to the image plane. We introduce here a technique involving, in two steps, two energy-minimization calculations. These calculations are done with the widely used and successfully tested Stillinger-Weber potential ${ }^{22}$ and with the parametrization of [23].

Far from the interface, the crystal structures of both grains are known and in particular the Si dumbbells correspond to atoms with two different altitudes $z$ along the $[1 \overline{1} 0]$ direction, common to both grains. The crystal pe- 


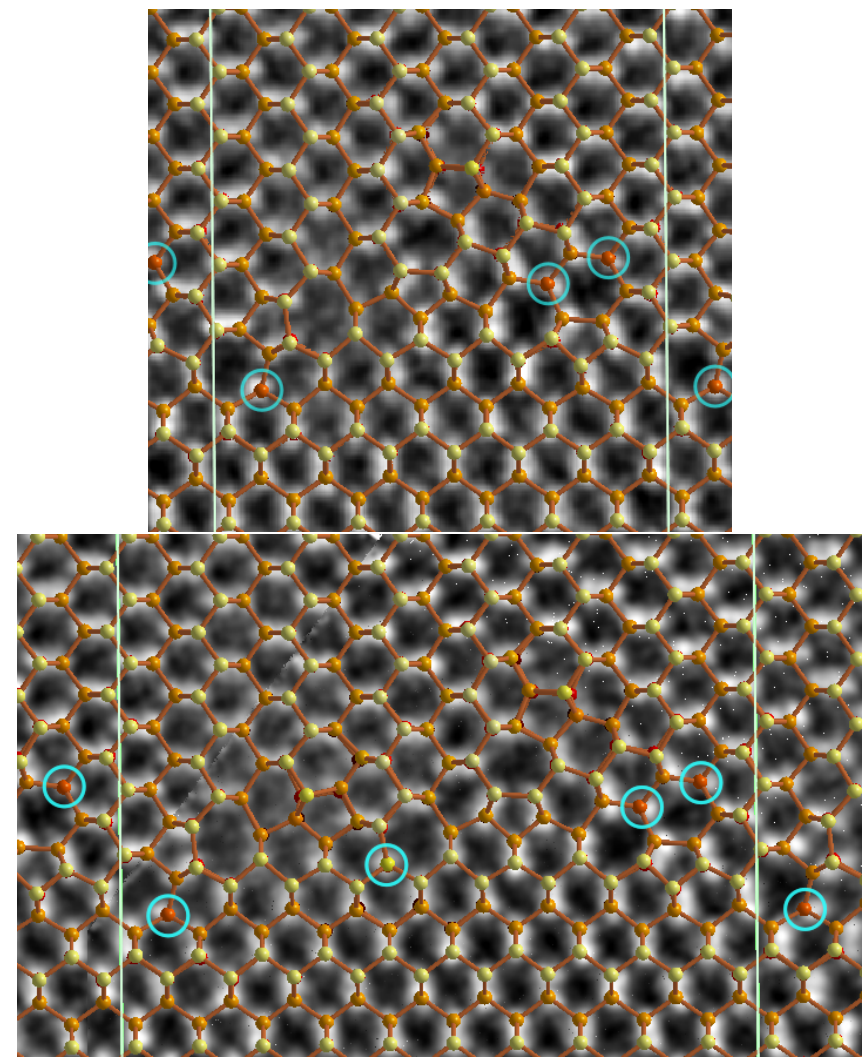

FIG. 4. (Color online) Electron microscopy images overlaid by the atomic configurations of the 5:7 and 10:7 approximants stable with the Stillinger-Weber interactions (respectively top and bottom images). For the bottom image, two images has been combined to get a sharper contrast. The light vertical lines indicate the periodicity along the interface. The coordinates $z$ perpendicular to the images are color coded from red (lower $z$ ) to yellow (higher $z$ ). All Si atoms are tetracoordinated. Atoms connected by bonds orthogonal to the images are surrounded by cyan circles.

riod along this direction is $s=a / \sqrt{2}$. We first assign a constant altitude $z=1 / 2 s$ to all atoms except for the $\mathrm{Si}$ dumbbells far enough from the interface to clearly belong to crystal sites. For these dumbbells, we set $z=1 / 4 s$ and $z=3 / 4 s$ to their respective two atoms. The isolated Si columns in Figure 3 correspond to atoms linked by bonds parallel to $[1 \overline{1} 0]$ in order to be tetrahedrally bonded. This is incompatible with a single-period structure along this direction and instead the fourfold coordination symmetry implies a doubling of the period. To get the initial atomic configuration, the atomic layers of two periods are therefore stacked together along $z$, resulting in a periodicity of $2 s$. Periodic boundary conditions are set along the $z$-direction and free surface boundary conditions are set for $x$ and $y$.

Doing a mere energy minimization starting from this configuration would be impossible because of the short inter-atomic distances resulting from the unrealistic identical $z$ value settings. The configuration would be unpredictably destroyed during the minimization. In a first step, we instead minimize the energy taking the $z$ coordinates as the only variables. Consequently, only the forces along $z$ are involved and they are initially zero by symmetry except for the few atoms in the crystal grains that have been given different $z$ values. At the beginning of the minimization, the surrounding of these few particularized atoms start to move, and then all the $z$ coordinates are progressively revealed. When the $z$ coordinates were first assigned, it made no difference which atom in a dumbbell is up and which atom is down as long as they are coherently displaced in the crystal. However, we have here two crystals and so we have a choice for the second grain once the first one is set. Considering the two possibilities, we have actually constructed two initial configurations that both have had their energy minimized with respect to the $z$ coordinates. Despite these two possibilities, only one correct configuration is obtained. Depending on the cases, either both configurations converge to the same final configuration, or one can be easily discarded because of a residual antiphase boundary inside one of the grains.

As a second step, a regular energy minimization with respect to all coordinates is performed. This regularizes the crystalline structure of the grains since the experimental $x$ and $y$ data where extracted with some random fluctuations around the equilibrium atomic positions.

With the result obtained from an entire experimental image, two slices are cut from the final atomic configuration. The first one corresponds to a 7:5 approximant, the second one to a 10:7 approximant. For each structure, the slice width is chosen to be its ideal periodicity $P$ introduced above (Eq 2). Subsequent energy minimizations are performed to adjust the atomic positions to the new boundary conditions, but now with periodic boundary conditions for the $x$ and $z$ directions and free surface boundary conditions for $y$. As shown in Figure 4, the final atomic configurations are in very good agreement with the corresponding parts of the experimental images. The coordinate files are available in Supporting Information $^{24}$. Our numerical method has been able to resolve the pentagon and heptagon structures as well as the connectivity of the atoms corresponding to individual spots in the images. All the atoms are now tetracoordinated.

\section{FIRST-PRINCIPLE CALCULATIONS}

\section{BigDFT calculations}

The approach presented in the previous section for recovering the atomic $z$ coordinates can in principle be done with first-principal calculations in the density functional theory (DFT) framework. This is not possible in this study because of the large number of atoms to consider. However, the interface structure is far from the regular lattice structure of silicon. For instance there are pentagonal and heptagonal Si loops instead of the 

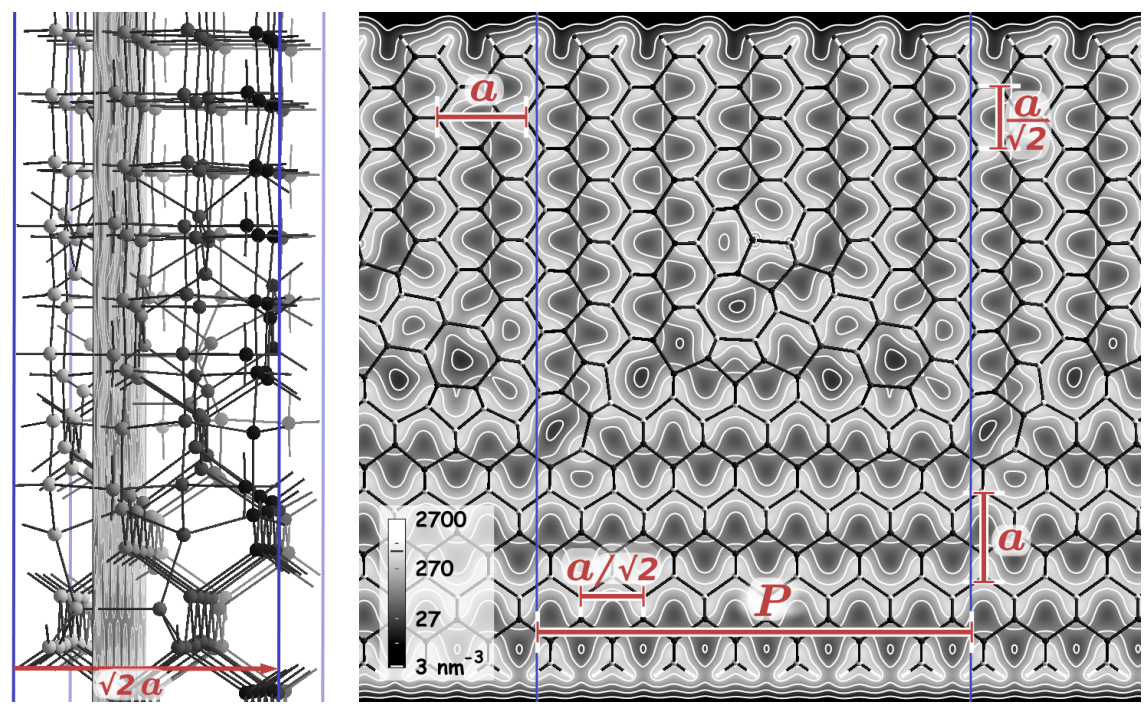

FIG. 5. Cross-section of the valence-electron density and ball-and-stick model of the 5:7 approximant. White and black in the grayscale represent respectively the highest and the lowest electron density. Left: Oblique view showing the location of the cross-section, which is perpendicular to the commensurate direction of the interface (common [110] direction). Along this direction, the periodicity of the structure is $\sqrt{2} a$, where $a$ is the cubic lattice parameter of silicon. Right: Plane view. The horizontal axis is the [001] direction of the upper grain and the [110] direction of the lower one. It is the opposite for the vertical axis. Along the incommensurate direction, $P$ is the periodicity of the approximant. The logarithmic scale of the density plot is indicated by the gray-scale bar. The average density is $N_{\mathrm{e}} / V=196 \mathrm{~nm}^{-3}$. The lattice parameter $a=547 \mathrm{pm}$ gives the length scale. The free-surfaces are passivated by hydrogen atoms.

usual hexagonal loops. Thus, to confirm the stability of the atomic configurations found with the effective interaction potential, we perform DFT calculations with the two approximants. This will also give us reliable interface energies. In this section, the configurations stable with Stillinger-Weber interactions are now the starting points for computing the electronic and atomic structures.

We have used BigDFT code ${ }^{17}$, which has the particularity of expressing the electronic wave functions with a set of wavelet basis functions. This characteristic makes it possible to control the computational precision in a systematic way, as with plane-wave basis functions, but furthermore gives a local decomposition of the wave functions. Besides the strong compression of the data and the excellent efficiency for parallel calculations both made possible by this locality, a variety of boundary conditions can be used, from isolated molecules to periodic crystals. This point is important here because we need to consider periodic conditions along $x$ and $z$, but free boundary conditions along $y$. Indeed, the configurations are periodic approximant along $x$, the direction $z$ of the interface is commensurate, and the $y$ free boundary conditions correspond to a material slab with surfaces parallel to the interface. As usual, the perturbation by these external surfaces on the bulk electronic structure is minimized here by terminating the dangling bonds with hydrogen atoms. For the same purpose, no surface reconstruction is considered and the external Si atoms are set on their perfect lattice sites. Keeping these atoms fixed on both surfaces would be possible but would preclude any relative global shift of the grains, which is an important parameter of the grain-boundary structures. Instead, free-bloc conditions are used, i.e., all $\mathrm{H}$ atoms and the $\mathrm{Si}$ atoms in the two outermost external atomic layers at each surface are fixed all together, but their center of gravity follows the resultant of the forces applied to them. This feature has been implemented in the BigDFT $\operatorname{code}^{25}$ for this study.

To check the independence of the results on the slab width, two different sizes along the $y$ direction are considered. In the 5:7 approximant there are $48 \mathrm{H}$ atoms and either 312 or $408 \mathrm{Si}$ atoms depending on the slab width. In the 10:7 approximant there are $68 \mathrm{H}$ atoms and either 440 or $576 \mathrm{Si}$ atoms. Pseudopotentials ${ }^{26,27}$ are used to simulate the core electrons. The Perdew-Burke-Ernzerhof (PBE) exchange correlation functional ${ }^{28}$ is used in the calculation. The choice of the code, of the pseudopotentials, and of the PBE functional has been assessed in a general study ${ }^{16}$ and gives high precision results. The $k$-point grid for calculations with the $\mathrm{Si}$ cubic cell is a $4 \times 4 \times 4$ Monkhorst Pack mesh. A $6 \times 4 \times 6$ mesh has been used for the orthogonal cell with paramerters $a / \sqrt{2}$, $a$ and $a / \sqrt{2}$ (see Fig. 1). The real-space grid spacing between the wavelet-function centers, $h_{\text {grid }}$, is set to $21 \mathrm{pm}$ corresponding to a high length resolution. With these conditions, the calculated value of the stable Si lattice parameter is $0.5466 \mathrm{~nm}$ in agreement with the nowadays DFT results ${ }^{16}$. For the grain-boundary calculations, the model periodicity along the $z$ axis corresponds to two [1 10$]$ periods and thus three $k$-points are used in this direction. Because of the large value of the periodicity along $x$ and the free-boundary conditions along $y$, no grid 
of $k$-points is necessary for both of these axes.

The density functional calculations consist in minimizing the energy with respect to both the atomic positions and the electronic structure. The energy minimization is stopped when forces on the atoms are lower than $10 \mathrm{meV} / \AA$. In the final configurations, all the Si atoms at the interface have kept their covalent bonding. The electronic density of the relaxed 7:5 approximant is shown in figure 5 and the coordinates of both approximants are available in Supporting Information ${ }^{24}$.

The interface energy $\gamma_{\mathcal{I}}$ is estimated by removing the other energy contributions from the total potential energy $E_{\text {pot }}$ of the configurations: bulk energy $\epsilon_{\mathrm{Si}}$, the residual elastic energies due to the residual strains (Eq. 3),

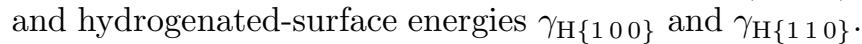
To calculate the surface energies we also consider slab geometries for each of the two grain orientations (see appendix and its figure 11). Along $y$, the thickness of the slabs are similar to those of the grain-boundary configurations. Free surfaces are hydrogenated too. Complete density functional calculations are done for all slabs. Now by considering the same geometry for each grain, but with additional atomic layers to thicken the slabs, we can extract the bulk energy from their total energy differences. This way, we finally get three estimates of the bulk energy, one for each of the grain orientations plus one calculated merely from the cubic cell. The maximum discrepancy between them is only $2 \mathrm{meV}$ per atom. This procedure is actually most useful to obtain all together bulk energy plus elastic energies of the slightly constrained grains. Indeed, the elastic constrains on the slabs are particular, with fixed strains along $x$ and $z$ (corresponding to imposed periodic conditions) and null stress along $y$ (free-bloc conditions). Taking into account these auxiliary calculations, the computed interface energies $\gamma_{\mathcal{I}}$ are $0.652 \mathrm{~J} / \mathrm{m}^{2}$ and $0.667 \mathrm{~J} / \mathrm{m}^{2}$, respectively for the $7: 5$ and 10:7 approximants. The discrepancy between the thickest and thinnest slabs is less then $0.007 \mathrm{~J} / \mathrm{m}^{2}$. An estimate of $\gamma_{\mathcal{I}}$ for the infinite incommensurate grain boundary, and not approximants, will be given in sectionVI and table I.

Another quantity, the adhesion energy $\gamma_{\mathrm{A}}$ is the gain per surface unit between the energy $E_{\text {pot }}$ of the configuration and the energy it would have if the two grains would be separated. So here, the higher energy $\gamma_{\mathrm{A}}$, the more stable is the interface. In our case, this last energy is deduced from slab calculations with a clean free surface on one side and hydrogenated surface on the other side. No complex surface reconstructions are taken into account, except the inter-plane relaxations, which are direct outputs of the calculation. The adhesion energy $\gamma_{\mathrm{A}}$ is equal to $3.26 \mathrm{~J} / \mathrm{m}^{2}$ for the $7: 5$ approximant. Though very close, the gain corresponds to a lower energy than the reported value $\gamma_{\mathrm{A}}=3.2 \mathrm{~J} / \mathrm{m}^{2}$ for an alternate structure of the $7: 5$ approximant $^{9}$. For the 10:7 approximant, $\gamma_{\mathrm{A}}$ is found equal to $3.21 \mathrm{~J} / \mathrm{m}^{2}$. The energy $\gamma_{\mathrm{A}}$ for separating the grains can be compared to the energies $4.65 \mathrm{~J} / \mathrm{m}^{2}$ and $3.38 \mathrm{~J} / \mathrm{m}^{2}$ obtained in this work for cleaving Si crystal at

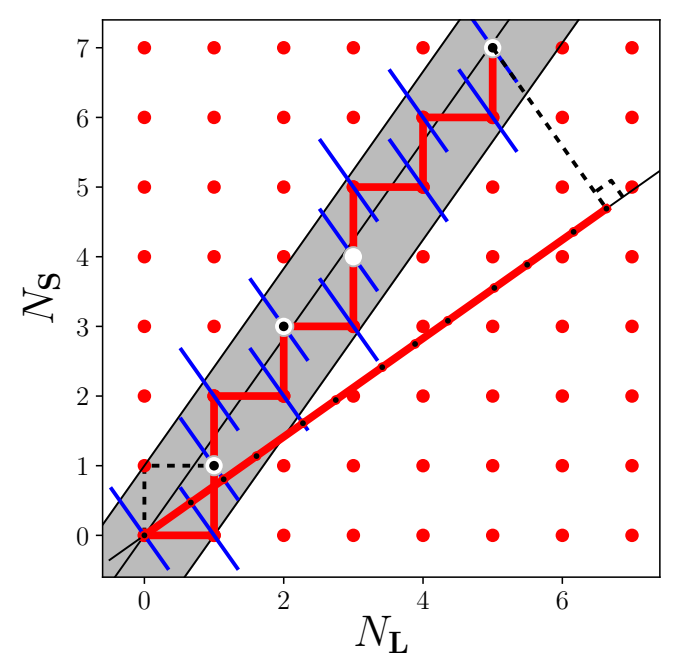

FIG. 6. Strip and projection method to generate a $\sqrt{2}$-tiling from the square lattice. The gray strip has a slope equal to $\sqrt{2}$ fixing the ratio $N_{\mathbf{S}} / N_{\mathbf{L}}=\sqrt{2}$. The lower limit of the strip goes through the point $(1,0)$ and the upper limit through $(0,1)$. The strip is half-bounded with the upper limit excluded here. The square edges and the sites of the square lattice inside the strip are selected. Once projected onto a straight line, they correspond respectively to the tiles and vertices of the tiling. If projected onto a line of slope $\sqrt{2}$ identical to the slope of the strip, the length of the segments would be in the ratio $P_{\mathbf{L}} / P_{\mathbf{S}}=1 / \sqrt{2}$. To get the ratio $P_{\mathbf{L}} / P_{\mathbf{S}}=\sqrt{2}$, they are projected onto a line of slope $1 / \sqrt{2}$. The equivalence with the cut method is also sketched: a line segment is periodically set on every site of the lattice (for clarity only those selected by the strip are drawn). The vertices of the tiling are now the intersection points between all segments and the line of slope $\sqrt{2}$ going through the origin. To get the correct length ratio, the segments are perpendicular to the line with slope $1 / \sqrt{2}$. The common length of the segments is the width of the strip along this direction. The white disks inside the strip correspond to the best rational approximations $N_{\mathbf{S}} / N_{\mathbf{L}}$ of $\sqrt{2}$, i.e. $\left|N_{\mathbf{S}} / N_{\mathbf{L}}-\sqrt{2}\right|<|p / q-\sqrt{2}|, \forall q \leq N_{\mathbf{L}}$. Among them, those with a central black dot correspond to the continued-fraction convergents, which are solutions of the stronger condition Eq 1.

(1 000$)$ and (1 10$)$ surfaces respectively. These values are similar to those found in [9].

\section{FROM APPROXIMANTS TO A COMPLETE DESCRIPTION OF THE INTERFACE}

\section{Hyper-space description and substitution rules}

The Fibonacci tiling, or Fibonacci word, is a prototype of $1 \mathrm{D}$ quasicrystals ${ }^{29}$. It is regularly presented to illustrate the strip and projection method ${ }^{30,31}$, the cut $\operatorname{method}^{32,33}$ or the inflation-rule method ${ }^{12,29,34}$. These methods generate or describe the quasicrystalline structures. The Fibonacci tiling is closely related to the golden 


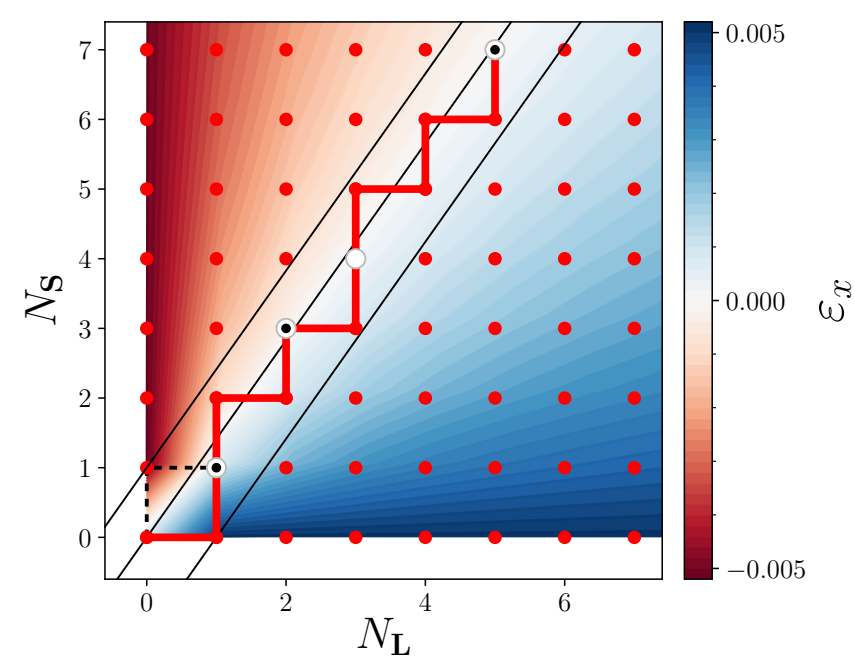

FIG. 7. Density plot of the strain $\varepsilon_{x, I I}$ in grain II for sequences made of $N_{\mathbf{L}}$ tiles $\mathbf{L}$ and $N_{\mathbf{S}}$ tiles $\mathbf{S}$ due to the misfit between the periodicities along the incommensurate direction of the two crystalline grains (see equations Eq. 3). Strain $\varepsilon_{x, I}$ in grain I is the opposite. The red line (bold polygonal curve) represents $\mathbf{S}_{3}=$ LSSLSLSSLSLS (Eqs. 4), a sequence that minimizes the strain field locally and on average.

ratio, i.e. the irrational number $\varphi=(1+\sqrt{5}) / 2$. Here, to combine together replicas of the two approximants described in the previous sections and get an incommensurate grain boundary, we derive similar properties for a tiling now based on $\sqrt{2}$ and associated with the silver ratio $\delta_{S}=1+\sqrt{2}$ instead of the golden ratio $\varphi$. To comply with the usual notations and broaden the scope of the discussion, we denote $\mathbf{S}=[7: 5]$ and $\mathbf{L}=[10: 7]$ the two approximants now regarded as structural units, with respective numbers $N_{\mathbf{S}}$ and $N_{\mathbf{L}}$ in a given sequence ( $\mathbf{S}$ for small and $\mathbf{L}$ for large).

First of all, why a grain boundary should be associated with such a tiling related to $\sqrt{2}$ ? The answer is given by equations 3 . They show that the sequence of structural units should maintain, locally and globally, the ratio $n_{s} / n_{\ell}$ the closest as possible to $\sqrt{2}$ in order to minimize the strain and thus the stress in the grains. It can be easily tested that for a sequence of $N_{\mathrm{S}}$ and $N_{\mathrm{L}}$ units with $N_{\mathrm{S}}+N_{\mathrm{L}} \rightarrow \infty$, the limit of $n_{s} / n_{\ell}=\left(7 N_{\mathbf{S}}+10 N_{\mathbf{L}}\right) /\left(5 N_{\mathbf{S}}+7 N_{\mathbf{L}}\right)$ is $\sqrt{2}$ when the limit of $N_{\mathbf{S}} / N_{\mathbf{L}}$ is itself $\sqrt{2}$. The lengths $P_{\mathbf{L}}$ and $P_{\mathbf{S}}$ of the two units are identified with their periods along $x$ (see Eqs. 2) and the ratio $P_{\mathbf{L}} / P_{\mathbf{S}}$ is equal to $\sqrt{2}$.

The strip and projection method is a method of choice to generate an incommensurate tiling (or sequence) of $\mathbf{S}$ and $\mathbf{L}$ units. To get a $\sqrt{2}$-tiling, a strip of slope $\sqrt{2}$ is used to select points of coordinates $\left(N_{\mathbf{L}}, N_{\mathbf{S}}\right)$ from a $2 \mathrm{D}$ simple square lattice. This is illustrated in figure 6 with the strip derived from the elementary lattice cell. This correspond to a very simple algorithm using the deviation $\Delta$ introduced in Eq. (1). Starting from no units, we add them one by one with the following rule: from $N_{\mathbf{S}}$ and $N_{\mathbf{L}}$ units, add one new $\mathbf{S}$ unit if $\Delta\left[N_{\mathbf{S}}+1, N_{\mathbf{L}}\right]<1$, if not add a $\mathbf{L}$ unit. In the last case, the numbers satisfied the relation $\Delta\left[N_{\mathbf{S}}, N_{\mathbf{L}}+1\right] \leq \sqrt{2}$. These two inequalities are linked to the strong condition for the best rational approximation seen in section II. Fig. 7 shows the relation between the strip-method and the residual strain due to the lattice misfit at the interface.

In these $\sqrt{2}$ tilings, the units can be grouped into larger tiles $\mathbf{L}_{\mathbf{1}}$ and $\mathbf{S}_{\mathbf{1}}$, which have a length ratio of $\sqrt{2}$ too. This gives the substitution rules:

$$
\begin{aligned}
& \mathbf{L} \rightarrow \mathbf{L}_{1} \equiv \mathbf{S L S} \\
& \mathbf{S} \rightarrow \mathbf{S}_{1} \equiv \mathbf{L S}
\end{aligned}
$$

where, in the context of this article, $\mathbf{L}_{1}$ and $\mathbf{S}_{1}$ are periodic grain boundaries corresponding to juxtapositions of elementary periods of the grain boundaries $\mathbf{L} \equiv \mathbf{L}_{0}$ and $\mathbf{S} \equiv \mathbf{S}_{0}$. Grain boundary $\mathbf{S}_{1}$ corresponds to the convergent structure [17:12] and $\mathbf{L}_{1}$ to the semi-convergent structure [24:17]. Starting from an initial unit and iterating the substitution rules (4) generates periodic tilings of increasing period. Equivalent by construction, an alternative method consists of using the recurrence relations

$$
\begin{aligned}
& \mathbf{L}_{n}=\mathbf{S}_{n-1} \mathbf{L}_{n-1} \mathbf{S}_{n-1} \\
& \mathbf{S}_{n}=\mathbf{L}_{n-1} \mathbf{S}_{n-1}
\end{aligned}
$$

and generating two sets of sequences in parallel. Since at step zero the ratio $P_{\mathbf{L}} / P_{\mathbf{S}}$ of the tile lengths is $\sqrt{2}$, these relations show that the length ratio of $\mathbf{L}_{n}$ and $\mathbf{S}_{n}$ is still $\sqrt{2}$ for any generation $n$, and that these lengths increase by a factor $\delta_{S}=1+\sqrt{2}$ after each substitution. The original substitution rules leading to the Fibonacci tiling are $\mathbf{L} \rightarrow \mathbf{L S}$ and $\mathbf{S} \rightarrow \mathbf{L}$, with the transformation of the numbers $N_{\mathbf{S}}$ and $N_{\mathbf{L}}$ from one generation to the other strongly linked to the golden ratio $\varphi$. For the $\sqrt{2}$-tiling, the matrix $\mathbf{M}$ that relates the column vector $\left(N_{\mathbf{L}} ; N_{\mathbf{S}}\right)$ before and after one substitution is

$$
M=\left(\begin{array}{ll}
1 & 1 \\
2 & 1
\end{array}\right)
$$

The two eigenvalues of $\mathbf{M}$ are the silver ratio $\delta_{S}=$ $1+\sqrt{2}$ and its conjugate $1-\sqrt{2}=-1 / \delta_{S}$. Since they are the solutions of a quadratic equation, i.e. the characteristic equation $(1-x)^{2}-2=0$, with a module greater than unity for the first one and smaller than unity for the second one, the eigenvalue $\delta_{S}$ is a Pisot number. This property is coherent with the fact that we are constructing a quasicrystalline tiling ${ }^{34-36}$. The vector $(1, \sqrt{2})$ is an eigenvector of $\mathbf{M}$ corresponding to the largest eigenvalue $\delta_{S}$. Therefore the ratio $N_{\mathbf{S}} / N_{\mathbf{L}}$ and thus the ratio $n_{s} / n_{\ell}$ tend to $\sqrt{2}$ when the number of successive substitutions tends to $\infty$. Thus, in both grains and away from the interface, the residual strain due to the lattice parameter mismatch actually tends to zero [see Eq. (3)].

Note 1. The $\sqrt{2}$-sequence described in this work is very closely related to the octonacci sequence ${ }^{37-40}$ :

$$
\mathbf{L}^{\prime} \rightarrow \mathbf{S}^{\prime} \mathbf{L}^{\prime} \mathbf{L}^{\prime} \quad \text { and } \quad \mathbf{S}^{\prime} \rightarrow \mathbf{L}^{\prime}
$$



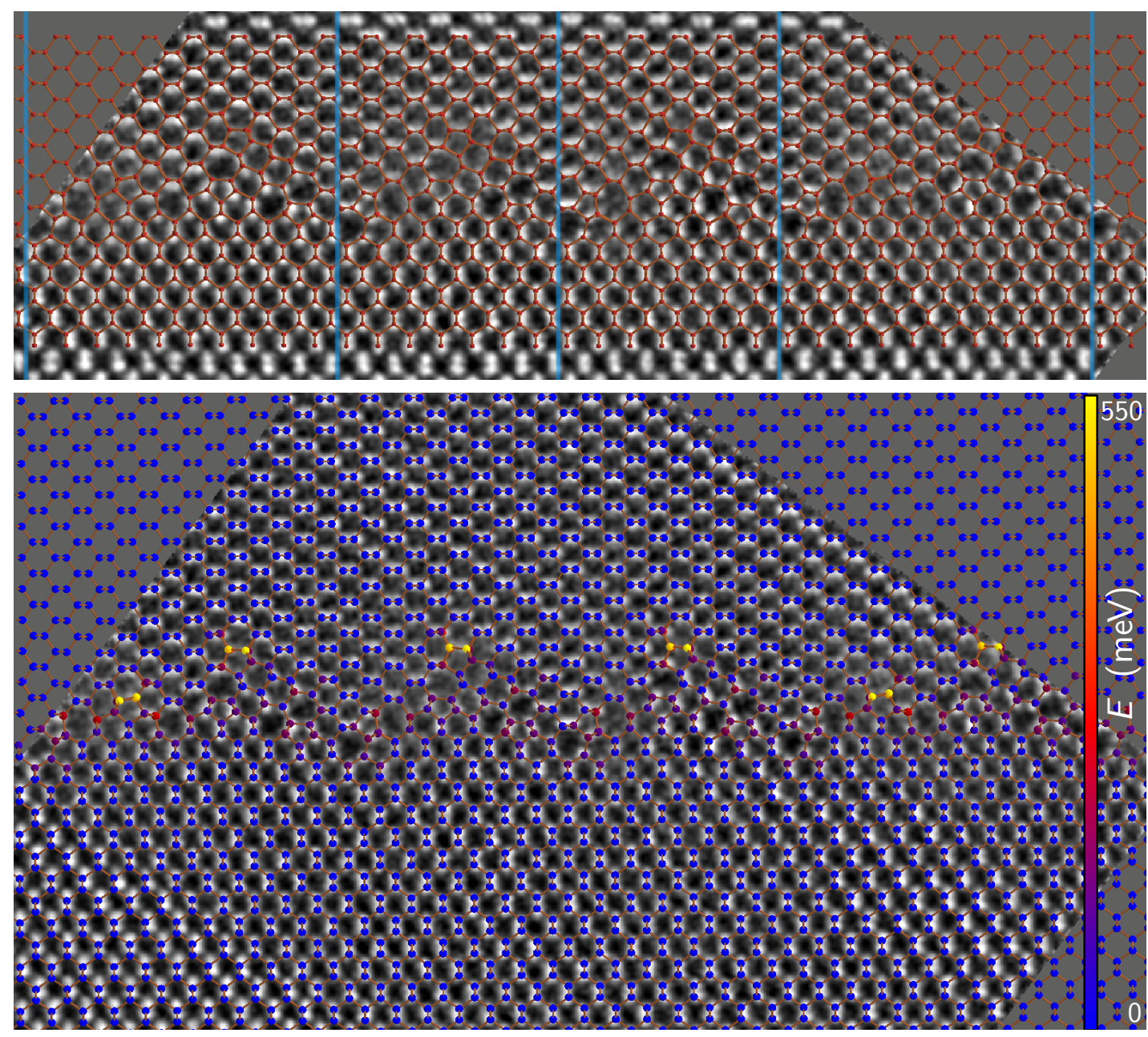

FIG. 8. Top: Overlay of the experimental image by a sequence of $\mathbf{S}$ and $\mathbf{L}$ tiles, i.e. respectively the [7:5] and [10:7] approximants, each one calculated by DFT. The vertical lines indicate the tile boundaries. Bottom: Overlay of the experimental image by a [41:29] approximant corresponding to the sequence $\mathbf{S}_{2}$, now relaxed with the Stillinger-Weber potential ${ }^{23}$. The energy of the atoms with this potential is color coded (online version) from blue (bulk energy) to red and yellow (highest energy). Note that the image contrast is correlated with the atom energies: when a high energy has been found the contrast is lower, probably indicating structure perturbations like a higher vacancy concentration or larger atomic moves.

(different orders in the tile concatenations in ref. 37, 39, and 40). Indeed, both sequences have the recurrence relation

$$
\mathbf{S}_{n}=\mathbf{S}_{n-2} \mathbf{S}_{n-1} \mathbf{S}_{n-1}
$$

deduced here from the equations $(5 \mathrm{a})$ and $(5 \mathrm{~b})$. One sequence is related to the other by choosing $\mathbf{L}^{\prime}=\mathbf{L S}$ and $\mathbf{S}^{\prime}=\mathbf{S}$. So, getting one or the other is a matter of the choice of the two basic tiles, $\mathbf{L}$ in the $\sqrt{2}$-sequence being smaller than $\mathbf{L}^{\prime}$. Since the elementary blocks $\mathbf{L}$ and $\mathbf{S}$ are directly extracted from the electron micrographs, the $\sqrt{2}$-sequence appears as a natural choice here.

Note 2. For simplicity in the Fibonacci tiling, the length ratio of the $\mathbf{L}$ and $\mathbf{S}$ tiles are usually set to $\varphi$, i.e. the same value as the tile proportion $N_{\mathbf{L}} / N_{\mathbf{S}}$. That way, in the strip-and-projection method on a square $2 \mathrm{D}$ lattice, the strip selects the tiles and a projection orthogonal to it gives directly the tiles. Here, in the $\sqrt{2}$-tiling, the length ratio is fixed to $\sqrt{2}$ by the periodicity of the approximants $\mathbf{L}=[10: 7]$ and $\mathbf{S}=[7: 5]$ observed by microscopy, while their proportion $N_{\mathbf{L}} / N_{\mathbf{S}}$ is the inverse $1 / \sqrt{2}$ to minimize the strain in the Si crystals.. Thus, the strip and the direction of the projection are not orthogonal as shown in figure 6. Equivalently in the cut method, the segment motif of the 2D-unit cell is not orthogonal to the strip direction, as shown in figure 6 too. A similar result could be obtained from a rhombic $2 \mathrm{D}$-lattice.

Note 3. While the infinite quasicrystalline $\sqrt{2}$-tiling corresponds to the slope $\sqrt{2}$, finite sequences $\mathbf{L}_{n}$ or $\mathbf{S}_{n}$ can be obtained from the square 2D-lattice with slopes $N_{\mathbf{S}} / N_{\mathbf{L}}$. That way, the strip-and-projection method leads to periodic 1D-lattices with $\mathbf{L}_{n}$ or $\mathbf{S}_{n}$ as the unit cells. Since in our case, the tiles $\mathbf{L}$ and $\mathbf{S}$ are periodic approximants at the atomic scale, we can build atomistic structures with periodic boundary conditions along the interface, These structures are thus well suitable for 
TABLE I. Interfaces energies $\left(\mathrm{J} / \mathrm{m}^{2}\right)$ obtained in this work with DFT calculations and different parametrizations of the Stilling-Weber potential: $\gamma_{\mathcal{I},[7: 5]}, \gamma_{\mathcal{I},[10: 7]}$, and $\gamma_{\mathcal{I},[577: 408]}$, respectively, for the periodic interfaces [7:5], [10:7], and $[577: 408] ; \gamma_{\mathcal{I}}$ for the incommensurate interface. Note: to compute these energies the bulk terms have been removed, in particular the elastic energy of the periodic approximants. While $\gamma_{\mathcal{I},[7: 5]}<\gamma_{\mathcal{I}}<\gamma_{\mathcal{I},[10: 7]}$, the total energy is lower for the incommensurate interface, which has no long range elastic stress.

\begin{tabular}{lcccc}
\hline \hline Atomic interactions & $\gamma_{\mathcal{I},[7: 5]}$ & $\gamma_{\mathcal{I},[10: 7]}$ & $\gamma_{\mathcal{I},[577: 408]}$ & $\gamma_{\mathcal{I}}$ \\
\hline DFT & 0.652 & 0.667 & & \\
S-W - Stillinger-Weber & & & & \\
S-W - Vink et al. & & & & \\
S-W - Pizzagalli et al. ${ }^{41}$ & 1.038 & 0.953 & 0.939 & 0.937 \\
S-W & 1.019 & 1.064 & 1.058 & 1.056 \\
\hline \hline
\end{tabular}

simulations at the atomic level.

Note 4. From Fig. 7 we can deduce that a random sequence of $\mathbf{L}$ and $\mathbf{S}$ units would increase the material strain and thus its elastic energy. This would also be the case, for instance, for sequences generated by the rules $\mathbf{L} \rightarrow$ LSLSL and $\mathbf{S} \rightarrow$ SLSS whose substitution matrix has the same eigenvectors than matrix $\mathbf{M}$ (Eq. 6) leading to the same tile ratio $N_{\mathbf{S}} / N_{\mathbf{L}}=\sqrt{2}$. However its largest eigenvalue, $3+\sqrt{2}$, is not a Pisot number; the smallest, $3-\sqrt{2}$, is larger than unity. As a consequence ${ }^{34-36}$, the extension perpendicular to the strip diverges in the square-lattice of Fig 7 and would thus correspond to a material with a larger strain.

\section{Application of the substitution rules}

With the recurrence rules and the atomistic configurations of the structural units, large models of the grain boundary can now be generated. Figure 8 shows part of a 41:29-approximant overlaying the experimental image of figure 2 in a very satisfactory way (sequence LSSLS equivalent to $\mathbf{S}_{2}$ by periodicity). These larger models can be easily relaxed with the Stillinger-Weber potential ${ }^{23}$, which we started with in section IV. The agreement is satisfactory too and figure 8 shows a correlation between the highest atomic energies and the fuzziness of the experimental spots.

The interface energy $\gamma_{\mathcal{I}}$ of the incommensurate grain boundary can now be estimated too (see appendix). Indeed, neglecting the correlation effects between structural units, the interface energies per 2D unit cell of the 7:5 and 10:7 approximants (section $\mathrm{V}$ ) can be weighted by their respective concentrations, $\sqrt{2} / \delta_{S}$ and $1 / \delta_{S}$. The 2D-unit cell areas $\sqrt{2} a P_{7: 5}$ and $\sqrt{2} a P_{10: 7}$ are averaged with the same respective weights (the $z$ periodicity, $\sqrt{2} a$, corresponds to two [1 10$]$ periods because of the interface reconstruction; the $x$ periodicity corresponds to $P$ in equation 2). Dividing the weighted energy average by the weighted area average yields to $\gamma_{\mathcal{I}}=0.660 \mathrm{~J} / \mathrm{m}^{2}$. Calculated in the same way, the calculated adhesion energy of the incommensurate grain boundary is $\gamma_{\mathrm{A}}=3.23 \mathrm{~J} / \mathrm{m}^{2}$.

For reference, we also have computed the interface energy $\gamma_{\mathcal{I}}$ obtained with the Stillinger-Weber potential ${ }^{23}$. This value, as well as the value obtained with the original parametrization by Stillinger and $\mathrm{Weber}^{22}$ are given in table I. A recent potential parametrization ${ }^{41}$ has also been tested and the result is included in table I. Since much larger configurations can be considered using Stillinger-Weber interactions than by DFT calculations, the interface energy of the 577:408 approximant has also been calculated for these interatomic potentials. This approximant corresponds to the sequence $S_{5}$ made of 41 $S$ and $29 L$ units. It has a period $P$ equal to $221.6 \mathrm{~nm}$ along the incommensurate direction. This gives the opportunity to check the validity of partitioning the whole grain boundary into $S$ and $L$ units to compute the interface energy, while neglecting the correlation effects. Indeed, the relative difference is only $0.2 \%$ between $\gamma_{\mathcal{I}}$ and $\gamma_{\mathcal{I},[577: 408]}$ taking into account long range elastic stresses as discussed in ${ }^{15}$ (see table I). With the large 577:408 approximant and the Stillinger-Weber potential ${ }^{23}$, we can also test the energy difference resulting from alternative sequences of $S$ and $L$ units. We have tested a random sequence of $41 S$ and $29 L$ and the extreme case where the $S$ and $L$ units are segregated. In both cases, the energy minimizations lead to a deformation of the layer due to the strain. The interface is not flat anymore and the buckling is larger for the segregated sequence than for the random one. To avoid this artifact, the minimization has been redone with constrains. The $y$ and $z$ coordinates of the first layer at each surface have been fixed, while the $x$ coordinates were unconstrained to let the strain field adjust to the $S$ and $L$ stacks along $x$. The incommensurate sequence has the lowest energy, while the random and the segregated ones have a surplus of $1 \mathrm{~mJ} / \mathrm{m}^{2}$ and $7 \mathrm{~mJ} / \mathrm{m}^{2}$, respectively. These results are coherent with the strain analysis illustrated in figure 7 . The random and the segregated sequences have excursions far from the ideal $S$ and $L$ concentrations, the segregated one being the extreme case.

\section{Hull plots}

Sharing the same cubic misorientation than the present $\mathrm{Si}$ grain boundary, the $90^{\circ}\langle 110\rangle$ tilt grain boundary in gold is therefore also an incommensurate grain boundary. For gold, it has been shown that this grain boundary has a frictionless - or superglide - property ${ }^{20,43,44}$. Such a property was first introduced for a unidimensional theoretical model of atoms in a periodic potential field ${ }^{45,46}$. Then, it was theoretically studied and experimentally found in several studies on friction ${ }^{42,43,47-61}$.

The frictionless property is unlikely for this silicon interface, because of its reconstruction extension with faceting clearly visible on the calculated-structure image 


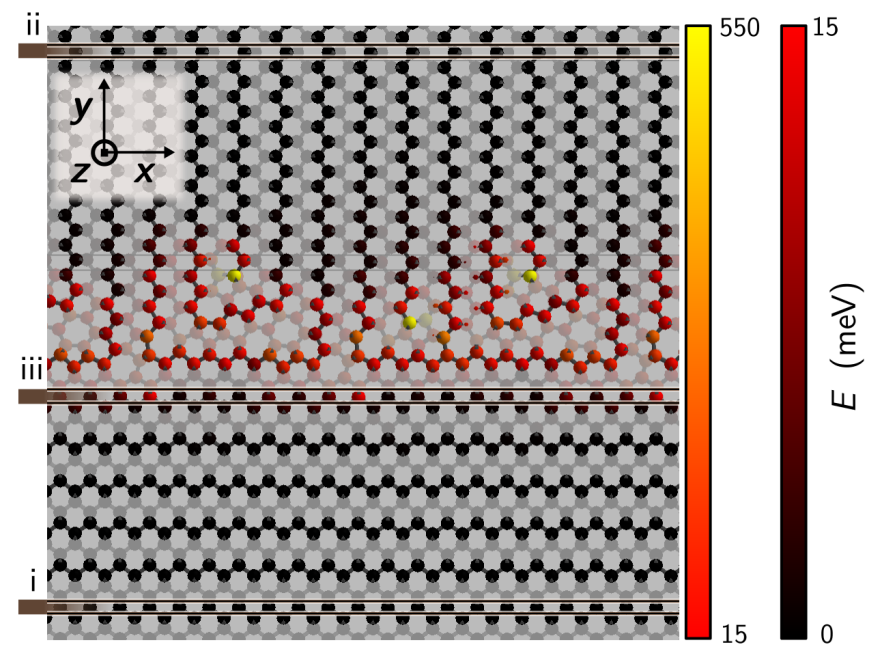

FIG. 9. Atomic-configuration visualization highlighting the calculation of the hull functions $\Delta x$ and $\Delta y$ plotted in figure 10. Only one atomic layer perpendicular to $z$ is not shaded by a transparent mask. Far from the interface, the atoms selected by the two lowest horizontal lines are representative of a crystalline atomic row, $i$, of grain I. In the same way, the two highest horizontal lines correspond to an atomic row, ii, of grain II. At the interface, the horizontal lines correspond to an atomic row, iii, that has been chosen for calculating its hull plots. Any atomic row could be selected, however this one is the closest to the interface with atoms having the same first-neighbor environment than those of grain I. That way, the influence of grain II on grain I can be analyzed. The opposite would be possible by choosing an atomic row in grain II. To simplify the discussion, row $i$ has been chosen to select crystallographically equivalent lattice sites than those of row iii. The atomic configuration has been relaxed with the Stillinger-Weber potential ${ }^{23}$ to get atomic energies. These energies are color coded with two successive color gradients. From zero to $15 \mathrm{meV}$, the first scale shows the energy range in rows iii. From $15 \mathrm{meV}$ to $550 \mathrm{meV}$, the second scale highlights the high energy spots at the interface.

(figure 9) as well as on the experimental images (figure 2 and in references [5, 6, and 19]). However, Aubry hullfunction analysis ${ }^{45,62}$ is the way to get a clear signature of this dynamical property from the static structure of an incommensurate atomic configuration. The hull function characterizes the atomic relaxation from a periodic lattice when the atoms are subject to a periodic potential having a different and incommensurate periodicity. Depending on the strength of the interactions, two regimes exist separated by an Aubry transition corresponding to a "breaking of analyticity" 45 when going from the unpinned states to the pinned one. Since the original unidimensional Frenkel-Kontorova case, the hull function has been generalized to more complex systems ${ }^{20,51,63}$.

At a grain boundary, each crystal row parallel to the incommensurate direction is perturbed by the other crystal and we consider the displacements of the atoms from their ideal lattices positions. Thus, we need these lattices positions, and a reference can be selected far from
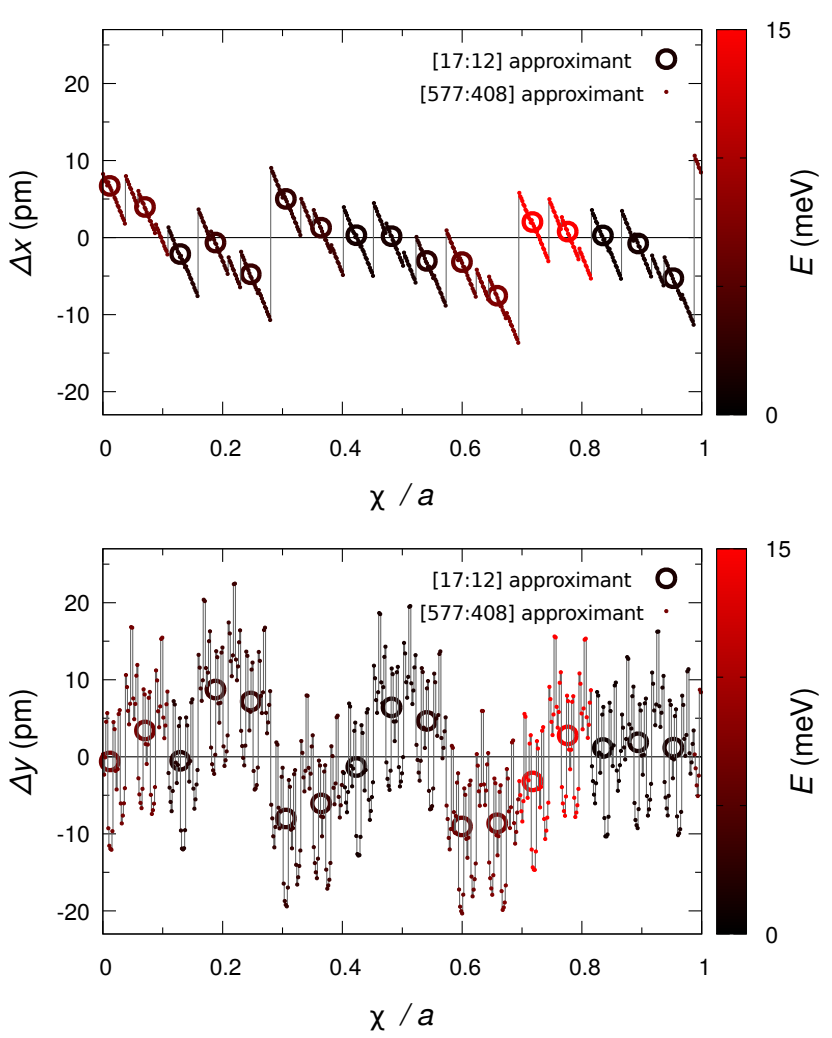

FIG. 10. Hull plots of the first atomic row of grain I at the incommensurate interface (see row iii of the lower grain in figure 9). The lattice parameter $a$ is also the periodicity $\ell$ of grain II. Top: atomic relaxation $\Delta x$ along the incommensurate direction. Bottom: relaxation $\Delta y$ perpendicular to the interface. The $\Delta y$ origin corresponds to the average of the $y$ coordinates in row iii. These hull plots are calculated with $17 \mathrm{Si}$ atoms of the [17:12] approximant (large circles) and 577 atoms of the [577:408] approximant (small dots). Function discontinuities cannot be revealed by inspecting the [17:12] results. However, note that the atomic relaxations of the [17:12] approximant are representative of those of the [577:408] one, the small-model data following the trends of the large-model ones. The discontinuities and the apparent fuzziness of the [577:408] curves are intrinsic to this type of incommensurate interface. The curves could be continuous for another material, but here they are discontinuous everywhere. It is a pinned-state characteristic ${ }^{42}$.

the interface. This is illustrated in figure 9 where rows $i$ and ii are representative of the lattice grain I and II respectively.

To represent the hull modulation function $\Delta x$ (see figure 10), the atomic displacements are plotted versus the place of their ideal sites relative to the perturbation, i.e. the other lattice periodicity. In grain I with period $s$ along $x$, the ideal coordinate of an atom with a lattice index $j \in \mathbb{Z}$, is $x_{0 j}=j s+\alpha$. The shift $\alpha$ is the $x$ coordinate of one arbitrary atom of row $i$, the origin being set here on one arbitrary atom of row ii. The relaxation displacement of an atom in row iii, i.e. its modulation from the ideal lattice site, is $\Delta x_{j}=x_{j}-x_{0 j}$. This dis- 
placement $\Delta x_{j}$ is plotted versus $\chi_{j}=\left(x_{0 j}\right.$ modulo $\left.\ell\right)$, where $\ell$ is the periodicity of grain II. The system being invariant by a shift $\ell$ of grain I because of the grain II periodicity, the modulo function wraps the complete relaxation behavior of the atoms in a function defined on $\left[0, \ell\left[\right.\right.$. The relaxation $\Delta y_{j}$ perpendicular to the interface versus $\chi_{j}$ is also plotted in figure 10. Here, $\Delta y_{j}=y_{j}-\bar{y}$, where $\bar{y}$ is the average of the $y_{j}$ in row iii. Using a lattice reference rather than $\bar{y}$ for calculating $\Delta y_{j}$ is also possible and would only result in a shift of the origin for $\Delta y_{j}$.

The discontinuous nature of the hull functions is clearly visible in figure 10. An infinitesimal glide of grain I relative to grain II, would correspond to an infinitesimal change of the coordinates $x_{0}$ and consequently to the quantity $\chi_{j}(\in[0, \ell[)$. Discontinuities of $\Delta x(\chi)$ would lead to finite jumps of the atomic displacements, and thus the system would have to overcome energy barriers. Therefore, this Si grain boundary has a pinned behavior and not a superglide one. The "broken analyticity" is particularly visible in the $\Delta y$ hull plot.

\section{CONCLUSION}

A general description of an incommensurate grain boundary in silicon has been given. While this paper is focused on a precise structure, it illustrates a general methodology for semi-conductors different from the analysis of incommensurate boundaries in metals ${ }^{20,42,43}$. This differences are mainly related to the behavior of the atomic bindings. Because of the covalent bonds, the boundary here is a quasicrystalline sequence of two elementary units with opposite internal strains: the 7:5 approximant $\mathbf{S}$ and the 10:7 approximant $\mathbf{L}$. First, these units have been selected since they are present in the experimental electron-microscopy images. Second, the unit $\mathbf{S}$ corresponds to the ratio $7 / 5$, one of the first best rational approximants of $\sqrt{2}$, while the unit $\mathbf{L}$ is a kind of complementary unit to $\mathbf{S}$ because of its almost opposite residual strain. Unit $\mathbf{L}$ corresponds to the semiconvergent ratio $10 / 7$ of $\sqrt{2}$. So, in the material, these two units can be combined together in a suitable way to reduce the strain and stress resulting from the incommensurate periodicities of the crystals on both sides of the boundary. For instance, concatenating $\mathbf{L}$ with $\mathbf{S}$ gives LS, which corresponds to the next best rational approximant $17 / 12$.

For both elementary units, we have conducted a detailed analysis of the 2D-images obtained by electron microscopy. A pattern recognition technique has been applied on high resolution images and has given reliable in-plane coordinates of the atomic spots. Taking advantage of the three components of color coding, the three correlation functions of the characteristic patterns - two orthogonal dumbbells and one single spot - can be combined together to give a comprehensive image of the grain-boundary. The three-dimensional atomic co- ordinates have been resolved with atomic-forces calculations using a Stilling-Weber potential and a two-step method. A first energy minimization is conducted, restricted only to the atomic coordinates perpendicular to the plane view. Once the third coordinates are recovered, the second step is an usual minimization that rectifies coherently all the coordinates of the structure. Finally, the structure has been refined with electronic structure calculations. The resulting $\mathbf{S}$ and $\mathbf{L}$ structures are fourfoldcoordinated silicon networks and are perfectly periodic in the two dimensions of the boundary.

To give a complete atomic description of the incommensurate boundary, the elementary units should form a sequence that reduces the interface stress. With tools adapted for quasicrystals, the atomic structures of $\mathbf{S}$ and $\mathbf{L}$ units can be combined together. In particular, we have given inflation rules related to the silver ratio and suitable for $\mathbf{S}$ and $\mathbf{L}$ tilings. Si atoms are still fourfold-coordinated in the resulting interfaces.

Suitable for incommensurate structures, the hull function of the grain boundary has been calculated, thanks to the large systems that can be constructed with the inflation rules. It demonstrates that no hypofriction property can be expected here.

This paper describes a structure corresponding to a perfect incommensurate interface minimizing the residual stress in the grains. However, defects of this ideal structure should be present in real materials, depending on the elaboration process. This is indeed what has been observed by electron microscopy and a rich variety of situations can be imagined. It is however out of the scope of this article to describe them here.

\section{ACKNOWLEDGMENTS}

The authors thank Alain Marty for useful discussions and suggestions.

\section{SUPPLEMENTAL MATERIAL}

The atomic coordinates of the $\mathbf{S}$ and $\mathbf{L}$ units are available as Supplemental Material ${ }^{24}$. The files are in plain ascii format and their content are self-explanatory. All coordinates are in $\AA$ unit and given in a Cartesian system. Besides the comment lines, the first two lines give the computer-box lattice parameters $\mathbf{a}, \mathbf{b}$, and $\mathbf{c}$ on the form $a_{x}, b_{x}, b_{y}, c_{x}, c_{y}$, and $c_{z}$, while $a_{y}=a_{z}=b_{z}=0$. Then, the following lines give for each atom the coordinates $x, y, z$, and the element name.

\section{Appendix: Interface-energy calculation}

To extract the interface energy $\gamma_{\mathcal{I}}$ of a grain boundary from the total energy $E_{\text {total }}$ of a configuration made of two crystalline grains, one must separately compute the 


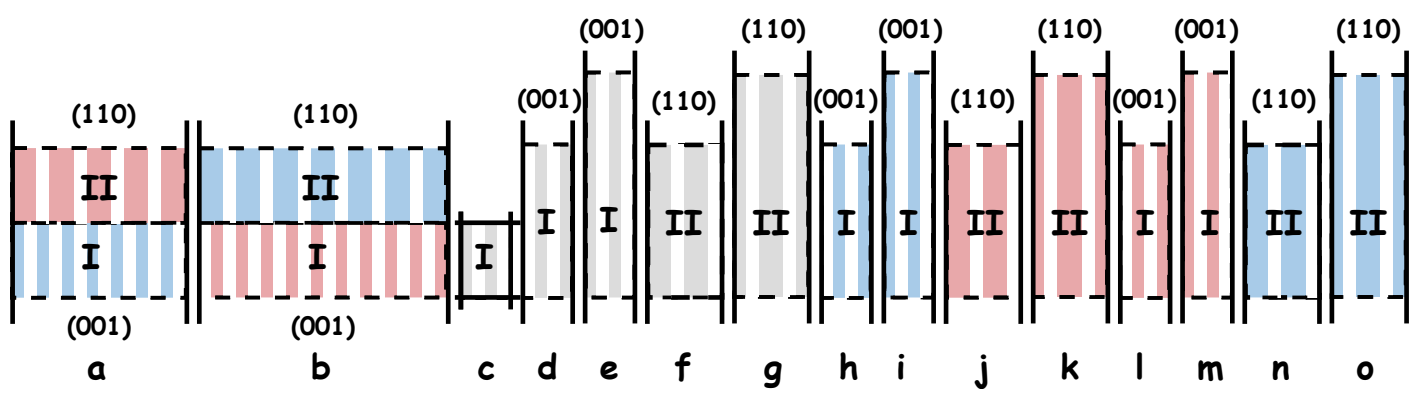

FIG. 11. Schematic view of the configurations constructed with grains I and II to compute the interface energy $\gamma_{\mathcal{I}}$. The solid lines represent the periodic boundary conditions. The dashed lines show the interfaces and the free surfaces of types (001) or (110). Configurations $\mathrm{a}$ and $\mathrm{b}$ are the 7:5 and 10:7 approximants, respectively. The tensile and compressive residual strains are shown in blue and red colors, respectively. These strains are due to the incommensurability between grain I and grain II periodicities. For taking into account their effects on the elastic energy and on the surface energies, these strains have been applied to configurations $\mathrm{h}$ to o. More precisely, the strains present in the 7:5 approximant (configuration a) have been used in configurations $\mathrm{h}$ to $\mathrm{k}$, while those of the 10:7 approximant (configuration b) have been used in l to o. The different layer widths, for instance between configurations $\mathrm{n}$ and $\mathrm{o}$, allow us to separately determine the strained-bulk energy and the strained-surface energy. Indeed, only the numbers of bulk atoms are different, while the free-surface areas are identical. Configuration c is a simple crystalline cell used to compute the reference bulk energy. Without applied strain too, configurations d to g provide us with two other ways to compute the bulk energy, as well as the values of the surface energies $\gamma_{\{001\}}$ and $\gamma_{\{110\}}$.

bulk energy $\epsilon_{\text {atom }}$ and the free surfaces ones $\gamma_{\text {surfaces }}$ to remove their contributions from $E_{\text {total }}$ :

$$
\gamma_{\mathcal{I}} S_{\mathcal{I}}=E_{\text {total }}-N_{\text {total }} \epsilon_{\text {atom }}-\gamma_{\text {surfaces }} S_{\mathcal{I}}
$$

where $S_{\mathcal{I}}$ is the area of the grain boundary and $N_{\text {total }}$ is the number of atoms in the configuration. In this paper, we determine $\gamma_{\mathcal{I}}$ of an incommensurate grain boundary from the contributions of its periodic grain-boundary units. These units are slightly constrained due to the incommensurate periodicities of the grains, while the incommensurate grain boundary itself has no intrinsic long range strain. Thus, to carefully calculate $\gamma_{\mathcal{I}}$, we must generalize the previous equation. The configurations taken into account are shown schematically in figure 11. For instance, from the total energy $E_{\text {total }}$ of the 7:5 con- figuration ("a" in figure 11) we calculate :

$$
\begin{aligned}
\gamma_{\mathcal{I},[7: 5]} S_{\mathcal{I},[7: 5]} & =E_{\text {total }} \\
& -N_{\mathrm{I}} \epsilon_{\mathrm{h}, \mathrm{i}}-N_{\mathrm{II}} \epsilon_{\mathrm{j}, \mathrm{k}} \\
& -\gamma_{\mathrm{h}, \mathrm{i}} S_{\mathcal{I},[7: 5]}-\gamma_{\mathrm{j}, \mathrm{k}} S_{\mathcal{I},[7: 5]}
\end{aligned}
$$

where $\epsilon_{\mathrm{h}, \mathrm{i}}$ and $\gamma_{\mathrm{h}, \mathrm{i}}$ are the strained bulk and surface energies calculated from configurations " $\mathrm{h}$ " and " $\mathrm{i}$ " in figure 11. Similarly, $\epsilon_{\mathrm{j}, \mathrm{k}}$ and $\gamma_{\mathrm{j}, \mathrm{k}}$ are derived from configurations "j" and "k". Area $S_{\mathcal{I},[7: 5]}$ is the interface area of the 7:5 unit. In the same way, $\gamma_{\mathcal{I},[10: 7]}$ can be calculated with the energies of configurations "b" and "l" to "o".

Finally, taking into account the respective frequencies $c_{\mathrm{S}}=\sqrt{2} / \delta_{S}$ and $c_{\mathrm{L}}=1 / \delta_{S}$ of units $\mathbf{S}=7: 5$ and $\mathbf{L}=$ $10: 7$, the interface energy of the incommensurate grain boundary is

$$
\gamma_{\mathcal{I}}=\frac{c_{S} \gamma_{\mathcal{I},[7: 5]} S_{\mathcal{I},[7: 5]}+c_{L} \gamma_{\mathcal{I},[10: 7]} S_{\mathcal{I},[10: 7]}}{c_{S} S_{\mathcal{I},[7: 5]}+c_{L} S_{\mathcal{I},[10: 7]}}
$$

* Frederic.Lancon@cea.fr

$\dagger$ Jean-Luc.Rouviere@cea.fr

${ }^{1}$ Frank Fournel, Hubert Moriceau, Bernard Aspar, Karine Rousseau, Joël Eymery, Jean-Luc Rouvière, and Noël Magnea, "Accurate control of the misorientation angles in direct wafer bonding," Applied Physics Letters 80, 793795 (2002).

2 A. Sakai, "Characterization of wafer-bonded substrates for advanced channels in Si-based MOSFET," in 2010 10th IEEE International Conference on Solid-State and Integrated Circuit Technology (2010) pp. 1517-1520.

3 E. Toyoda, A. Sakai, O. Nakatsuka, H. Isogai, T. Senda, K. Izunome, M. Ogawa, and S. Zaima, "Characteriza- tion of bonding structures of directly bonded hybrid crystal orientation substrates," Thin Solid Films 517, 323 - 326 (2008), fifth International Conference on Silicon Epitaxy and Heterostructures (ICSI-5).

${ }^{4}$ Eiji Toyoda, Akira Sakai, Hiromichi Isogai, Takeshi Senda, Koji Izunome, Kazuhiko Omote, Osamu Nakatsuka, and Shigeaki Zaima, "Characterization and analyses of interface structures in directly bonded $\mathrm{Si}(011) / \mathrm{Si}(001)$ substrates," Japanese Journal of Applied Physics 48, 021208 (2009).

5 J-L Rouvière, F Lançon, K Rousseau, D Caliste, P-H Jouneau, and F Fournel, "Structure of an incommensurate 90 degrees Si grain boundary resolved with the help of 
a Cs-corrector for illumination," Journal of Physics: Conference Series 209, 012041 (2010).

6 T. Signamarcheix, B. Biasse, A.-M. Papon, E. Nolot, F. Mazen, J. Leveneur, O. Faynot, L. Clavelier, and B. Ghyselen, "Crystallographic orientation engineering in silicon-on-insulator substrates," Applied Physics Letters 96, 262111 (2010).

7 T. Kato, Y. Nakamura, J. Kikkawa, A. Sakai, E. Toyoda, K. Izunome, O. Nakatsuka, S. Zaima, Y. Imai, S. Kimura, and O. Sakata, "Structural change of direct silicon bonding substrates by interfacial oxide out-diffusion annealing," Thin Solid Films 518, S147 - S150 (2010), sixth International Conference on Silicon Epitaxy and Heterostructures.

8 Chun-Yung Sung, Haizhou Yin, H. Y. Ng, K. L. Saenger, V. Chan, S. W. Crowder, Jinghong Li, J. A. Ott, R. Bendernagel, J. J. Kempisty, Victor Ku, H. K. Lee, Zhijiong Luo, A. Madan, R. T. Mo, P. Y. Nguyen, G. Pfeiffer, M. Raccioppo, N. Rovedo, D. Sadana, J. P. de Souza, Rong Zhang, Zhibin Ren, and C. H. Wann, "High performance cmos bulk technology using direct silicon bond (dsb) mixed crystal orientation substrates," in IEEE International Electron Devices Meeting, 2005. IEDM Technical Digest. (2005) pp. 225-228.

9 Hiroaki Kariyazaki, Tatsuhiko Aoki, Koji Izunome, and Koji Sueoka, "Molecular simulation on interfacial structure and gettering efficiency of direct silicon bonded (110)/(100) substrates," Journal of Applied Physics 107, 113509 (2010).

10 Nicolas Rivier, "A botanical quasicrystal," J. Phys. Colloques 47, C3-299-C3-309 (1986).

11 Denis Gratias and A. Thalal, "Hidden symmetries in general grain boundaries," Philosophical Magazine Letters 57, 63-68 (1988).

12 Adrian P. Sutton, "Irrational tilt grain boundaries as onedimensional quasicrystals," Acta metall. 36, 1291-1299 (1988).

13 P. A. Deymier, M. Shamsuzzoha, and J. D. Weinberg, "Experimental evidence for a structural unit model of quasiperiodic grain boundaries in aluminium," Journal of Materials Research 6, 1461-1468 (1991).

14 Adrian P. Sutton, "Irrational interfaces," Progress in Materials Science 36, 167-202 (1992).

15 Adrian P. Sutton and R. W. Balluffi, Interfaces in Crystalline Materials (Oxford University Press, New York, 1995).

16 Kurt Lejaeghere, Gustav Bihlmayer, Torbjoern Bjoerkman, Peter Blaha, Stefan Bluegel, Volker Blum, Damien Caliste, Ivano E. Castelli, Stewart J. Clark, Andrea Dal Corso, Stefano de Gironcoli, Thierry Deutsch, John Kay Dewhurst, Igor Di Marco, Claudia Draxl, Marcin Dulak, Olle Eriksson, Jose A. Flores-Livas, Kevin F. Garrity, Luigi Genovese, Paolo Giannozzi, Matteo Giantomassi, Stefan Goedecker, Xavier Gonze, Oscar Granaes, E. K. U. Gross, Andris Gulans, François Gygi, D. R. Hamann, Phil J. Hasnip, N. A. W. Holzwarth, Diana Iusan, Dominik B. Jochym, François Jollet, Daniel Jones, Georg Kresse, Klaus Koepernik, Emine Kuecuekbenli, Yaroslav O. Kvashnin, Inka L. M. Locht, Sven Lubeck, Martijn Marsman, Nicola Marzari, Ulrike Nitzsche, Lars Nordstrom, Taisuke Ozaki, Lorenzo Paulatto, Chris J. Pickard, Ward Poelmans, Matt I. J. Probert, Keith Refson, Manuel Richter, Gian-Marco Rignanese, Santanu Saha, Matthias Scheffler, Martin Schlipf, Karlheinz Schwarz, Sangeeta Sharma, Francesca Tavazza, Patrik Thunstroem,
Alexandre Tkatchenko, Marc Torrent, David Vanderbilt, Michiel J. van Setten, Veronique Van Speybroeck, John M. Wills, Jonathan R. Yates, Guo-Xu Zhang, and Stefaan Cottenier, "Reproducibility in density functional theory calculations of solids," Science 351, aad3000 (2016).

17 Luigi Genovese, Alexey Neelov, Stefan Goedecker, Thierry Deutsch, Seyed Alireza Ghasemi, Alexander Willand, Damien Caliste, Oded Zilberberg, Mark Rayson, Anders Bergman, and Reinhold Schneider, "Daubechies wavelets as a basis set for density functional pseudopotential calculations," The Journal of Chemical Physics 129, 014109 (2008).

18 Frédéric Lançon, Luigi Genovese, and Joël Eymery, "Towards simulation at picometer-scale resolution: Revisiting inversion domain boundaries in GaN," Phys. Rev. B 98, 165306 (2018).

19 N. Cherkashin, O. Kononchuk, S. Reboh, and M. Hÿtch, "Application of the O-lattice theory for the reconstruction of the high-angle near 90 degree tilt $\mathrm{Si}(110) /(001)$ boundary created by wafer bonding," Acta Materialia 60, 1161 - 1173 (2012).

20 A. Gautam, C. Ophus, F. Lançon, V. Radmilovic, and U. Dahmen, "Atomic structure characterization of an incommensurate grain boundary," Acta Materialia 61, 5078 - 5086 (2013).

21 S. Paciornik, R. Kilaas, J. Turner, and U. Dahmen, "A pattern recognition technique for the analysis of grain boundary structure by HREM," Ultramicroscopy 62, 15 -27 (1996).

22 Frank H. Stillinger and Thomas A. Weber, "Computer simulation of local order in condensed phases of silicon," Phys. Rev. B 31, 5262-5271 (1985).

23 R.L.C. Vink, G.T. Barkema, W.F. van der Weg, and Normand Mousseau, "Fitting the stillinger-weber potential to amorphous silicon," Journal of Non-Crystalline Solids 282, 248 - 255 (2001).

24 See Supplemental Material at [URL will be inserted by publisher] for atomic-coordinate files ("ascii" files).

25 BigDFT, "version 1.8," (2016).

26 S. Goedecker, M. Teter, and J. Hutter, "Separable dualspace gaussian pseudopotentials," Phys. Rev. B 54, 17031710 (1996).

27 C. Hartwigsen, S. Goedecker, and J. Hutter, "Relativistic separable dual-space Gaussian pseudopotentials from $\mathrm{H}$ to Rn," Phys. Rev. B 58, 3641-3662 (1998).

28 John P. Perdew, Kieron Burke, and Matthias Ernzerhof, "Generalized gradient approximation made simple," Phys. Rev. Lett. 77, 3865-3868 (1996).

29 Dov Levine and Paul J. Steinhardt, "Quasicrystals. I. definition and structure," Phys. Rev. B 34, 596-616 (1986).

30 A. Katz and M. Duneau, "Quasiperiodic structures obtained by the projection method," Journal de Physique Colloques 47, C3-103-C3-112 (1986).

31 Masashi Torikai, Komajiro Niizeki, and Takashi Odagaki, "Binary self-similar one-dimensional quasilattices: $\mathrm{Mu}$ tual local-derivability classification and substitution rules," Journal of the Physical Society of Japan 70, 2918-2933 (2001).

32 Per Bak, "Icosahedral crystals from cuts in six-dimensional space," Scripta Metallurgica 20, 1199 - 1204 (1986).

33 T. Janssen, "Aperiodic crystals: A contradictio in terminis?" Physics Reports 168, 55 - 113 (1988).

34 Frédéric Lançon. and Luc Billard, "Binary tilings and quasi-quasicrystalline tilings," Phase Transitions 44, 37- 
46 (1993).

35 C. Godrèche and F. Lançon, "A simple example of a nonPisot tiling with five-fold symmetry," Journal de Physique I 2, 207-220 (1992).

${ }^{36}$ C. Godrèche and J. M. Luck, "Indexing the diffraction spectrum of a non-pisot self-similar structure," Phys. Rev. B 45, 176-185 (1992).

37 Sire, Clément, Mosseri, Rémy, and Sadoc, Jean-François, "Geometric study of a 2D tiling related to the octagonal quasiperiodic tiling," J. Phys. France 50, 3463-3476 (1989).

38 Clément Sire, "Electronic spectrum of a 2D quasi-crystal related to the octagonal quasi-periodic tiling," EPL (Europhysics Letters) 10, 483 (1989).

${ }^{39}$ H. Q. Yuan, U. Grimm, P. Repetowicz, and M. Schreiber, "Energy spectra, wave functions, and quantum diffusion for quasiperiodic systems," Phys. Rev. B 62, 15569-15578 (2000).

40 Walter Steurer and Sofia Deloudi, "Tilings and coverings," in Crystallography of Quasicrystals: Concepts, Methods and Structures (Springer Berlin Heidelberg, Berlin, Heidelberg, 2009) pp. 7-47.

41 L Pizzagalli, J Godet, J Guénolé, S Brochard, E Holmstrom, K Nordlund, and T Albaret, "A new parametrization of the stillinger-weber potential for an improved description of defects and plasticity of silicon," Journal of Physics: Condensed Matter 25, 055801 (2013).

42 Frédéric Lançon, "Aubry transition in a real material: Prediction for its existence in an incommensurate gold/gold interface," Europhysics Letters 57, 74-79 (2002).

43 Frédéric Lançon, J.-M. Pénisson, and U. Dahmen, "Quasicrystalline gold interface with a frictionless property," Europhysics Letters 49, 603-609 (2000).

44 Frédéric Lançon, Jia Ye, Damien Caliste, Tamara Radetic, Andrew M. Minor, and Ulrich Dahmen, "Superglide at an internal incommensurate boundary," Nano Letters 10, 695-700 (2010).

45 Serge Aubry, "The new concept of transitions by breaking of analyticity in a crystallographic model," in Solitons and Condensed Matter Physics, Solid-State Sciences, Vol. 8, edited by A. R. Bishop and T. Schneider (Springer-Verlag, Berlin Heidelberg, 1978) pp. 264-277.

46 Pascal Quémerais, "Ground-states and growth structures for one-dimensional models with periodic to quasiperiodic transitions," in Quasicrystals - Current Topics, edited by Esther Belin-Ferré, Claire Berger, Marianne Quiquandon, and Anne Sadoc (World Scientific, Singapore, 2000) pp. $187-211$

47 J. E. Sacco and J. B. Sokoloff, "Free sliding in lattices with two incommensurate periodicities," Phys. Rev. B 18, 6549-6559 (1978)

48 Motohisa Hirano and Kazumasa Shinjo, "Atomistic locking and friction," Phys. Rev. B 41, 11837-11851 (1990).

49 Kazumasa Shinjo and Motohisa Hirano, "Dynamics of friction: superlubric state," Surface Science 283, 473-478 (1993).

50 Marek Cieplak, Elizabeth D. Smith, and Mark O. Robbins, "Molecular origins of friction: The force on adsorbed layers," Science 265, 1209-1212 (1994), http://www.sciencemag.org/cgi/reprint/265/5176/1209.pdf.

51 Michael Weiss and Franz-Josef Elmer, "Dry friction in the Frenkel-Kontorova-Tomlinson model: Static properties," Phys. Rev. B 53, 7539-7549 (1996).

52 M. R. Sørensen, K. W. Jacobsen, and P. Stoltze, "Simulations of atomic-scale sliding friction," Phys. Rev. B 53, 2101-2113 (1996).

53 Motohisa Hirano, Kazumasa Shinjo, Reizo Kaneko, and Yoshitada Murata, "Observation of superlubricity by scanning tunneling microscopy," Phys. Rev. Lett. 78, 14481451 (1997).

${ }^{54}$ Gang He, Martin H. Müser, and Mark O. Robbins, "Adsorbed layers and the origin of static friction," Science 284, 1650-1652 (1999).

55 Martin H. Müser and Mark O. Robbins, "Conditions for static friction between flat crystalline surfaces," Phys. Rev. B 61, 2335-2342 (2000).

56 J.S. Ko and A.J. Gellman, "Friction anisotropy at $\mathrm{Ni}(100) / \mathrm{Ni}(100)$ interfaces," Langmuir 16, 8343-8351 (2000).

57 Martin H. Müser, Michael Urbakh, and Mark O. Robbins, "Statistical mechanics of static and low-velocity kinetic friction," in Advances in Chemical Physics (John Wiley \& Sons, Ltd, 2003) Chap. 5, pp. 187-272.

58 Martin Dienwiebel, Gertjan S. Verhoeven, Namboodiri Pradeep, Joost W. M. Frenken, Jennifer A. Heimberg, and Henny W. Zandbergen, "Superlubricity of graphite," Phys. Rev. Lett. 92, 126101 (2004).

59 A. Socoliuc, R. Bennewitz, E. Gnecco, and E. Meyer, "Transition from stick-slip to continuous sliding in atomic friction: Entering a new regime of ultralow friction," Phys. Rev. Lett. 92, 134301 (2004).

60 Ali Erdemir and Jean-Michel Martin, eds., Superlubricity (Elsevier Science, 2007).

61 T. Brazda, A. Silva, N. Manini, A. Vanossi, R. Guerra, E. Tosatti, and C. Bechinger, "Experimental observation of the Aubry transition in two-dimensional colloidal monolayers," Phys. Rev. X 8, 011050 (2018).

62 Serge Aubry, "Devil's staircase and order without periodicity in classical condensed matter," Journal de Physique 44, 147-162 (1983).

63 Serge Aubry, Jean-Pierre Gosso, Gilles Abramovici, JeanLuc Raimbault, and Pascal Quemerais, "Effective discommensurations in the incommensurate ground states of the extended Frenkel-Kontorowa models," Physica D: Nonlinear Phenomena 47, 461 - 497 (1991). 